\title{
Successive-Cancellation Decoding of Binary Polar Codes Based on Symmetric Parametrization
}

\author{
Jun Muramatsu Senior Member, IEEE
}

\begin{abstract}
This paper introduces algorithms for the successive-cancellation decoding and the successive-cancellation list decoding of binary polar source/channel codes. By using the symmetric parametrization of conditional probability, we reduce both space and time complexity compared to the original algorithm introduced by Tal and Vardy.
\end{abstract}

\section{Index Terms}

binary polar codes, source coding with decoder side information, channel coding, successive-cancellation decoding

\section{INTRODUCTION}

Polar source/channel codes were introduced by Arıkan [1], [2], [3]. When these codes are applied to source coding with decoder side information for joint stationary memoryless sources, the coding rate achieves a fundamental limit called the conditional entropy. When applied to the channel coding of a symmetric channel, the coding rate achieves a fundamental limit called the channel capacity. Arıkan introduced successive-cancellation decoding, which can be implemented with computational complexity of $O\left(N \log _{2} N\right)$ where $N$ is block length.

In this paper, we introduce algorithms for successive-cancellation decoding and successive-cancellation list decoding based on the work of Tal and Vardy [10]. Our constructions can be applied to both polar source codes and polar channel codes. Furthermore, the proposed list decoding algorithm reduces the space and time complexity compared to [10].

\section{Definitions AND Notations}

Throughout this paper, we use the following definitions and notations.

For a given $n$, let $N \equiv 2^{n}$ denote the block length. We assume that the number $n$ is given as a constant, which means that all algorithms have access to this number. We use the bit-indexing approach introduced in [1]. The indexes of a $N$-dimensional vector are represented by $n$-bit sequences as $X^{N} \equiv\left(X_{0^{n}}, \ldots, X_{1^{n}}\right)$, where $0^{n} / 1^{n}$ denotes the $n$-bit all zero/one sequence. To represent an interval of integers, we use the following notations

$$
\begin{aligned}
{\left[0^{n}: b^{n}\right] } & \equiv\left\{0^{n}, \ldots, b^{n}\right\} \\
{\left[0^{n}: b^{n}\right) } & \equiv\left[0^{n}: b^{n}\right] \backslash\left\{b^{n}\right\} . \\
{\left[b^{n}: 1^{n}\right] } & \equiv\left[0^{n}: 1^{n}\right] \backslash\left[0^{n}: b^{n}\right) \\
\left(b^{n}: 1^{n}\right] & \equiv\left[0^{n}: 1^{n}\right] \backslash\left[0^{n}: b^{n}\right] .
\end{aligned}
$$

For a given subset $\mathcal{I}$ of $\left[0^{n}: 1^{n}\right]$, we define the sub-sequences of $X^{N}$ as

$$
X_{\mathcal{I}} \equiv\left\{X_{b^{n}}\right\}_{b^{n} \in \mathcal{I}} .
$$

Let $c^{l} b^{k} \in\{0,1\}^{l+k}$ be the concatenation of $b^{k} \in\{0,1\}^{k}$ and $c^{l} \in\{0,1\}^{l}$. For given $b^{k} \in\{0,1\}^{k}$ and $c^{l} \in\{0,1\}^{l}$, we define subsets $c^{l}\left[0: b^{k}\right]$ and $c^{l}\left[0: b^{k}\right)$ of $\{0,1\}^{k+l}$ as

$$
\begin{aligned}
c^{l}\left[0: b^{k}\right] & \equiv\left\{c^{l} d^{k}: d^{k} \in\left[0^{k}: b^{k}\right]\right\} \\
c^{l}\left[0: b^{k}\right) & \equiv\left\{c^{l} d^{k}: d^{k} \in\left[0^{k}: b^{k}\right)\right\} .
\end{aligned}
$$

The bipolar-binary conversion, $\mp_{b}$, of $b \in\{0,1\}$ is defined as

$$
\mp_{b} \equiv \begin{cases}- & \text { if } b=1 \\ + & \text { if } b=0\end{cases}
$$

J. Muramatsu is with NTT Communication Science Laboratories, NTT Corporation, 2-4, Hikaridai, Seika-cho, Soraku-gun, Kyoto, 619-0237 Japan (E-mail: jun.muramatsu@ieee.org). 


\section{BINARY POLAR CODES}

In this section, we revisit the binary polar source/channel codes introduced in previous works [1], [2], [3], [8]. Assume that $\{0,1\}$ is the binary finite field. For given positive integer $n$, polar transform $G$ is defined as

$$
G \equiv\left(\begin{array}{ll}
1 & 0 \\
1 & 1
\end{array}\right)^{\otimes n} \Pi_{\mathrm{BR}}
$$

where $\otimes n$ denotes the $n$-th Kronecker power and $\Pi_{\mathrm{BR}}$ is the bit-reversal permutation matrix [1]. Next, vector $\boldsymbol{u} \in\{0,1\}^{N}$ is defined as $\boldsymbol{u} \equiv \boldsymbol{x} G$ for given vector $\boldsymbol{x} \in\{0,1\}^{N}$. For completeness, an algorithm that computes $\boldsymbol{u}$ is given in Appendix $\mathrm{A}$ Let $\left\{\mathcal{I}_{0}, \mathcal{I}_{1}\right\}$ be a partition of $\left[0^{n}: 1^{n}\right]$, satisfying $\mathcal{I}_{0} \cap \mathcal{I}_{1}=\emptyset$ and $\mathcal{I}_{0} \cup \mathcal{I}_{1}=\left[0^{n}: 1^{n}\right]$. We define $\left\{\mathcal{I}_{0}, \mathcal{I}_{1}\right\}$ later.

Let $\boldsymbol{X} \equiv\left(X_{0^{n}}, \ldots, X_{1^{n}}\right)$ and $\boldsymbol{Y} \equiv\left(Y_{0^{n}}, \ldots, Y_{1^{n}}\right)$ be random variables and let $\boldsymbol{U} \equiv\left(U_{0^{n}}, \ldots, U_{1^{n}}\right)$ be a random variable defined as $\boldsymbol{U} \equiv \boldsymbol{X} G$. Then $P_{U_{\mathcal{I}_{0}} U_{\mathcal{I}_{1}} \boldsymbol{Y}}$, the joint distribution of $\left(U_{\mathcal{I}_{0}}, U_{\mathcal{I}_{1}}, \boldsymbol{Y}\right)$, is defined using the joint distribution $P_{\boldsymbol{X} \boldsymbol{Y}}$ of $(\boldsymbol{X}, \boldsymbol{Y})$ as

$$
P_{U_{\mathcal{I}_{0}} U_{\mathcal{I}_{1}} \boldsymbol{Y}}\left(u_{\mathcal{I}_{0}}, u_{\mathcal{I}_{1}}, \boldsymbol{y}\right) \equiv P_{\boldsymbol{X} \boldsymbol{Y}}\left(\left(u_{\mathcal{I}_{1}}, u_{\mathcal{I}_{0}}\right) G^{-1}, \boldsymbol{y}\right),
$$

where the elements in $\left(u_{\mathcal{I}_{1}}, u_{\mathcal{I}_{0}}\right)$ are sorted in index order before operation $G^{-1}$. We refer to $u_{\mathcal{I}_{1}}$ and $u_{\mathcal{I}_{0}}$ as frozen bits and unfrozen bits, respectively.

Let $P_{U_{b^{n}} \mid U_{\left[0^{n}: b^{n}\right)} \boldsymbol{Y}}$ be the conditional probability distribution, defined as

$$
P_{U_{b^{n}} \mid U_{\left[0^{n}: b^{n}\right)} \boldsymbol{Y}}\left(u_{b^{n}} \mid u_{\left[0^{n}: b^{n}\right)}, \boldsymbol{y}\right) \equiv \frac{\sum_{u_{\left(b^{n}: 1^{n}\right]}} P_{U_{\mathcal{I}_{0}} U_{\mathcal{I}_{1}} \boldsymbol{Y}}\left(u_{\mathcal{I}_{0}}, u_{\mathcal{I}_{1}}, \boldsymbol{y}\right)}{\sum_{u_{\left[b^{n}: 1^{n}\right]}} P_{U_{\mathcal{I}_{0}} U_{\mathcal{I}_{1}} \boldsymbol{Y}}\left(u_{\mathcal{I}_{0}}, u_{\mathcal{I}_{1}}, \boldsymbol{y}\right)}
$$

For vector $u_{\mathcal{I}_{1}}$ and side information $\boldsymbol{y} \in \mathcal{Y}^{N}$, output $\widehat{\boldsymbol{u}} \equiv \boldsymbol{f}\left(u_{\mathcal{I}_{1}}, \boldsymbol{y}\right)$ of successive-cancellation (SC) decoder $\boldsymbol{f}$ is defined recursively as

$$
\widehat{u}_{b^{n}} \equiv \begin{cases}f_{b^{n}}\left(\widehat{u}_{\left[0^{n}: b^{n}\right)}, \boldsymbol{y}\right) & \text { if } b^{n} \in \mathcal{I}_{0} \\ u_{b^{n}} & \text { if } b^{n} \in \mathcal{I}_{1}\end{cases}
$$

using function $\left\{f_{b^{n}}\right\}_{b^{n} \in \mathcal{I}_{0}}$ defined as

$$
f_{b^{n}}\left(u_{\left[0^{n}: b^{n}\right)}, \boldsymbol{y}\right) \equiv \arg \max _{u \in\{0,1\}} P_{U_{b^{n}} \mid U_{\left[0^{n}: b^{n}\right)} \boldsymbol{Y}}\left(u \mid u_{\left[0^{n}: b^{n}\right)}, \boldsymbol{y}\right),
$$

which is the maximum a posteriori decision rule after an observation $\left(u_{\left[0^{n}: b^{n}\right)}, \boldsymbol{y}\right)$.

For a polar source code (with decoder side information), $\boldsymbol{x} \in\{0,1\}^{N}$ is a source output, $u_{\mathcal{I}_{1}}$ is a codeword, and $\boldsymbol{y} \in \mathcal{Y}^{N}$ is a side information output. The decoder reproduces source output $\widehat{\boldsymbol{x}} \equiv \boldsymbol{f}\left(u_{\mathcal{I}_{1}}, \boldsymbol{y}\right) G^{-1}$ from codeword $u_{\mathcal{I}_{1}}$ and $\boldsymbol{y}$. The (block) decoding error probability is given as $\operatorname{Prob}\left(\boldsymbol{f}\left(U_{\mathcal{I}_{1}}, \boldsymbol{Y}\right) G^{-1} \neq \boldsymbol{X}\right)$.

For a systematic polar channel code [3], we define $\mathcal{I}_{0}^{\prime}$ and $\mathcal{I}_{1}^{\prime}$ as

$$
\begin{aligned}
& \mathcal{I}_{0}^{\prime} \equiv\left\{b_{0} b_{1} \cdots b_{n-1}: b_{n-1} \cdots b_{1} b_{0} \in \mathcal{I}_{0}\right\} \\
& \mathcal{I}_{1}^{\prime} \equiv\left\{b_{0} b_{1} \cdots b_{n-1}: b_{n-1} \cdots b_{1} b_{0} \in \mathcal{I}_{1}\right\}
\end{aligned}
$$

for given $\left(\mathcal{I}_{0}, \mathcal{I}_{1}\right)$. We assume that encoder and decoder share a vector $u_{\mathcal{I}_{1}}$. The encoder computes $\left(x_{\mathcal{I}_{1}^{\prime}}, u_{\mathcal{I}_{0}}\right)$ from message $x_{\mathcal{I}_{0}^{\prime}}$ and shared vector $u_{\mathcal{I}_{1}}$ so that $\left(x_{\mathcal{I}_{1}^{\prime}}, x_{\mathcal{I}_{0}^{\prime}}\right)=\left(u_{\mathcal{I}_{1}}, u_{\mathcal{I}_{0}}\right) G^{-1}$, where the elements in $\left(x_{\mathcal{I}_{1}^{\prime}}, x_{\mathcal{I}_{0}^{\prime}}\right)$ and $\left(u_{\mathcal{I}_{1}}, u_{\mathcal{I}_{0}}\right)$ are sorted in index order before operating $G^{-1}$. An algorithm for this computation is introduced in Appendix $\mathbb{B}$. The encoder then generates channel input $\boldsymbol{x} \equiv\left(x_{\mathcal{I}_{0}^{\prime}}, x_{\mathcal{I}_{1}^{\prime}}\right)$, where the elements in $\left(x_{\mathcal{I}_{1}^{\prime}}, x_{\mathcal{I}_{0}^{\prime}}\right)$ are sorted in index order. The decoder reproduces channel input $\widehat{\boldsymbol{x}} \equiv \boldsymbol{f}\left(u_{\mathcal{I}_{1}}, \boldsymbol{y}\right) G^{-1}$ from channel output $\boldsymbol{y} \in \mathcal{Y}^{N}$ and shared vector $u_{\mathcal{I}_{1}}$, where $\widehat{x}_{\mathcal{I}_{0}^{\prime}}$ is a reproduction of the message. The (block) decoding error probability is also given as $\operatorname{Prob}\left(\boldsymbol{f}\left(U_{\mathcal{I}_{1}}, \boldsymbol{Y}\right) G^{-1} \neq \boldsymbol{X}\right)$.

For a non-systematic polar channel code, $u_{\mathcal{I}_{0}}$ is a message and vector $u_{\mathcal{I}_{1}}$ is shared by the encoder and decoder. The encoder generates channel input $\boldsymbol{x} \in\{0,1\}^{N}$ as $\boldsymbol{x} \equiv\left(u_{\mathcal{I}_{1}}, u_{\mathcal{I}_{0}}\right) G^{-1}$, where the elements in $\left(u_{\mathcal{I}_{1}}, u_{\mathcal{I}_{0}}\right)$ are sorted in index order before operating $G^{-1}$. The decoder reproduces the pair of vectors $\left(u_{\mathcal{I}_{1}}, \widehat{u}_{\mathcal{I}_{0}}\right) \equiv \boldsymbol{f}\left(u_{\mathcal{I}_{1}}, \boldsymbol{y}\right)$ from channel output $\boldsymbol{y} \in \mathcal{Y}^{N}$ and shared vector $u_{\mathcal{I}_{1}}$, where $\widehat{u}_{\mathcal{I}_{0}}$ is a reproduction of the message. The (block) decoding error probability is given as $\operatorname{Prob}\left(\boldsymbol{f}\left(U_{\mathcal{I}_{1}}, \boldsymbol{Y}\right) \neq\left(U_{\mathcal{I}_{0}}, U_{\mathcal{I}_{1}}\right)\right)$.

We have the following lemmas.

Lemma 1 ([2. Theorem 2],[8] Theorem 4.10]): Define $\mathcal{I}_{0}$ as

$$
\mathcal{I}_{0} \equiv\left\{b^{n} \in\left[0^{n}: 1^{n}\right]: Z\left(U_{b^{n}} \mid U_{\left[0^{n}: b^{n}\right)}, Y_{\left[0^{n}: 1^{n}\right]}\right) \leq 2^{-2^{n \beta}}\right\}
$$

where $Z\left(U_{b^{n}} \mid U_{\left[0^{n}: b^{n}\right)}, Y_{\left[0^{n}: 1^{n}\right]}\right)$ is the source Bhattacharyya parameter introduced in [2]. Then we have

$$
\begin{aligned}
& \lim _{n \rightarrow \infty} \frac{\left|\mathcal{I}_{0}\right|}{2^{n}}=1-H(X \mid Y) \\
& \lim _{n \rightarrow \infty} \frac{\left|\mathcal{I}_{1}\right|}{2^{n}}=H(X \mid Y)
\end{aligned}
$$


for any $\beta \in[0,1 / 2)$.

Lemma 2 ([8. Proposition 2.7]):

$$
\operatorname{Prob}\left(f_{b^{n}}\left(U_{\left[0^{n}: b^{n}\right.}, \boldsymbol{Y}\right) \neq U_{b^{n}}\right) \leq Z\left(U_{b^{n}} \mid U_{\left[0^{n}: b^{n}\right)}, Y_{\left[0^{n}: 1^{n}\right]}\right) .
$$

We have the following lemma, which can be shown as in a previous proof [1].

Lemma 3 ([6. Lemma 2],[9. Eq. (1)]):

$$
\begin{aligned}
\operatorname{Prob}\left(\boldsymbol{f}\left(U_{\mathcal{I}_{1}}, \boldsymbol{Y}\right) G^{-1} \neq \boldsymbol{X}\right) & =\operatorname{Prob}\left(\boldsymbol{f}\left(U_{\mathcal{I}_{1}}, \boldsymbol{Y}\right) \neq\left(U_{\mathcal{I}_{0}}, U_{\mathcal{I}_{1}}\right)\right) \\
& \leq \sum_{b^{n} \in \mathcal{I}_{0}} \operatorname{Prob}\left(f_{b^{n}}\left(U_{\left[0^{n}: b^{n}\right)}, \boldsymbol{Y}\right) \neq U_{b^{n}}\right) .
\end{aligned}
$$

From the above lemmas, we have the fact that the rate of polar codes attains the fundamental limit and the decoding error probability goes to zero as $n \rightarrow \infty$. For example, we can obtain $\mathcal{I}_{0}$ by using the technique introduced in [7], [9] In the following sections, we assume that $\mathcal{I}_{0}$ is given arbitrary.

\section{SYMMETRIC PARAMETRIZATION}

In this section, we introduce the polar transform based on symmetric parametrization. Given $P_{U}$, a probability distribution of binary random variable $U$, let $\theta$ be defined as

$$
\theta \equiv P_{U}(0)-P_{U}(1)
$$

Then we have

$$
P_{U}(u)=\frac{1 \mp u}{2}
$$

where $\mp_{u}$ is the bipolar-binary conversion of $u$.

In the basic polar transform, a pair of binary random variables $\left(U_{0}, U_{1}\right)$ is transformed into

$$
\begin{aligned}
& U_{0}^{\prime} \equiv U_{0} \oplus U_{1} \\
& U_{1}^{\prime} \equiv U_{1},
\end{aligned}
$$

where $\oplus$ denotes the addition on the binary finite field. Assume that random variables $U_{0}, U_{1} \in\{0,1\}$ are independent. For each $i \in\{0,1\}$, let $\theta_{i}$ be defined as

$$
\theta_{i} \equiv P_{U_{i}}(0)-P_{U_{i}}(1)
$$

First, we have

$$
\begin{aligned}
P_{U_{0}^{\prime}}(0) & =P_{U_{0}}(0) P_{U_{1}}(0)+P_{U_{0}}(1) P_{U_{1}}(1) \\
& =\frac{1+\theta_{0}}{2} \cdot \frac{1+\theta_{1}}{2}+\frac{1-\theta_{0}}{2} \cdot \frac{1-\theta_{1}}{2} \\
& =\frac{1+\theta_{0} \theta_{1}}{2},
\end{aligned}
$$

where the first equality comes from the definition of $U_{0}^{\prime}$ and the fact that $U_{0}$ and $U_{1}$ are mutually independent. The above yields

$$
\begin{aligned}
P_{U_{0}^{\prime}}(1) & =1-P_{U_{0}^{\prime}}(0) \\
& =\frac{1-\theta_{0} \theta_{1}}{2} .
\end{aligned}
$$

From (2) and (3), we have

$$
P_{U_{0}^{\prime}}\left(u_{0}^{\prime}\right)=\frac{1 \mp u_{0}^{\prime} \theta_{0} \theta_{1}}{2},
$$

where $\mp u_{0}^{\prime}$ is the bipolar-binary conversion of $u_{0}^{\prime}$. Let $\theta_{0}^{\prime}$ be defined as

$$
\theta_{0}^{\prime} \equiv P_{U_{0}^{\prime}}(0)-P_{U_{0}^{\prime}}(1)
$$

From (2)-(5), we have

$$
\theta_{0}^{\prime}=\theta_{1} \theta_{0}
$$

It should be noted that, since symmetric parametrization is a binary version of the Fourier transform of the probability distribution [5, Definitions 24 and 25], the right hand side of (6) corresponds to the Fourier transform of the convolution.

We have

$$
P_{U_{0}^{\prime} U_{1}^{\prime}}\left(u_{0}^{\prime}, 0\right)=P_{U_{0}}\left(u_{0}^{\prime}\right) P_{U_{1}}(0)
$$




$$
\begin{aligned}
& =\frac{1 \mp u_{0}^{\prime} \theta_{0}}{2} \cdot \frac{1+\theta_{1}}{2} \\
& =\frac{1 \mp u_{0}^{\prime} \theta_{0} \theta_{1}+\theta_{1} \mp u_{0}^{\prime} \theta_{0}}{4},
\end{aligned}
$$

where the first equality comes from the definition of $U_{0}^{\prime}$ and $U_{1}^{\prime}$, and the fact that $U_{0}$ and $U_{1}$ are mutually independent. Then we have

$$
\begin{aligned}
P_{U_{1}^{\prime} \mid U_{0}^{\prime}}\left(0 \mid u_{0}^{\prime}\right) & =\frac{P_{U_{1}^{\prime} U_{0}^{\prime}}\left(0, u_{0}^{\prime}\right)}{P_{U_{0}^{\prime}}\left(u_{0}^{\prime}\right)} \\
& =\frac{1+\left[\theta_{1} \mp_{u_{0}^{\prime}} \theta_{0}\right] /\left[1 \mp{u_{0}^{\prime}}_{1} \theta_{1} \theta_{0}\right]}{2} \\
P_{U_{1}^{\prime} \mid U_{0}^{\prime}}\left(1 \mid u_{0}^{\prime}\right) & =1-P_{U_{1}^{\prime} \mid U_{0}^{\prime}}\left(0 \mid u_{0}^{\prime}\right) \\
& =\frac{1-\left[\theta_{1} \mp u_{0}^{\prime} \theta_{0}\right] /\left[1 \mp u_{0}^{\prime} \theta_{1} \theta_{0}\right]}{2} .
\end{aligned}
$$

Let $\theta_{1}^{\prime}$ be defined as

$$
\theta_{1}^{\prime} \equiv P_{U_{1}^{\prime} \mid U_{0}^{\prime}}\left(0 \mid u_{0}\right)-P_{U_{1}^{\prime} \mid U_{0}^{\prime}}\left(1 \mid u_{0}\right)
$$

From (8)-10), we have

$$
\begin{aligned}
\theta_{1}^{\prime} & =\frac{\theta_{1} \mp u_{0}^{\prime} \theta_{0}}{1 \mp u_{0}^{\prime} \theta_{1} \theta_{0}} \\
& =\frac{\theta_{1} \mp_{u_{0}^{\prime}} \theta_{0}}{1 \mp u_{0}^{\prime} \theta_{0}^{\prime}},
\end{aligned}
$$

where the second equality comes from (6).

\section{Successive-Cancellation Decoding}

This section introduces the algorithm of successive-cancellation decoding based on that introduced in [10].

We assume that Algorithms 13 have access to the number of transforms, $n$, the frozen bits $u_{\mathcal{I}_{1}}^{(n)}$, and the memory space

$$
\begin{aligned}
& \Theta \equiv\left\{\Theta[k]\left[c^{n-k}\right]: \begin{array}{rl}
k & \in\{0, \ldots, n\} \\
c^{n-k} & \in\left[0^{n-k}: 1^{n-k}\right]
\end{array}\right\} \\
& \mathrm{U} \equiv\left\{\begin{aligned}
k & \in\{0, \ldots, n\} \\
\mathrm{U}[k]\left[c^{n-k}\right][b]: c^{n-k} & \in\left[0^{n-k}: 1^{n-k}\right] \\
b & \in\{0,1\}
\end{aligned}\right\},
\end{aligned}
$$

where $\Theta[k]\left[c^{n-k}\right]$ is a real number variable, $\mathrm{U}[k]\left[c^{n-k}\right][b]$ is a binary variable, and $c^{0}$ denotes the null string. It should be noted that $\Theta$ has $\sum_{k=0}^{n} 2^{n-k}=2^{n+1}-1=2 N-1$ variables and $\mathrm{U}$ has $2 \sum_{k=0}^{n} 2^{n-k}=2^{n+2}-2=4 N-2$ variables.

In the following, we assume that $\left\{U_{c^{n}}^{(0)}\right\}_{c^{n} \in\left[0^{n}: 1^{n}\right]}$ is a memoryless source, that is, $P_{U_{\left[0^{n}: 1^{n}\right]}^{(0)}}$ is defined as

$$
P_{U_{\left[0^{n}: 1^{n}\right]}^{(0)}}\left(u_{\left[0^{n}: 1^{n}\right]}^{(0)}\right) \equiv \prod_{c^{n} \in\left[0^{n}: 1^{n}\right]} P_{U_{c^{n}}^{(0)}}\left(u_{c^{n}}^{(0)}\right),
$$

where $\left\{P_{U_{c^{n}}^{(0)}}\right\}_{c^{n} \in\left[0^{n}: 1^{n}\right]}$ is given depending on the context. It should be noted that $\left\{U_{c^{n}}^{(0)}\right\}_{c^{n} \in\left[0^{n}: 1^{n}\right]}$ is allowed to be nonstationary. We recursively define $U_{b^{n}}^{(n)}$ as

$$
\begin{aligned}
U_{c^{n-k} b^{k-1} 0}^{(k)} & \equiv U_{c^{n-k} 0 b^{k-1}}^{(k-1)} \oplus U_{c^{n-k} 1 b^{k-1}}^{(k-1)} \\
U_{c^{n-k} b^{k-1} 1}^{(k)} & \equiv U_{c^{n-k} 1 b^{k-1}}^{(k-1)}
\end{aligned}
$$

for given $b^{n} \in\{0,1\}^{n}$ and $c^{n-k} \in\{0,1\}^{n-k}$. This yields $U_{\left[0^{n}: 1^{n}\right]}^{(n)}=U_{\left[0^{n}: 1^{n}\right]}^{(0)} G$, which is the polar transform of $U_{\left[0^{n}: 1^{n}\right]}^{(0)}$. The goal of update $\Theta\left(\Theta, \mathrm{U}, n, \mathrm{~b}^{n}\right)$ at Line 3 of Algorithm 1 is to compute

$$
\theta_{b^{n}}^{(n)} \equiv P_{U_{b^{n}}^{(n)} \mid U_{\left[0^{n}: b^{n}\right)}^{(n)}}\left(0 \mid u_{\left[0^{n}: b^{n}\right)}^{(n)}\right)-P_{U_{b^{n}}^{(n)} \mid U_{\left[0^{n}: b^{n}\right)}^{(n)}}\left(1 \mid u_{\left[0^{n}: b^{n}\right)}^{(n)}\right)
$$

recursively starting from

$$
\theta_{b^{n}}^{(0)} \equiv P_{U_{b^{n}}^{(0)}}(0)-P_{U_{b^{n}}^{(0)}}(1)
$$


In Algorithm 2, we compute a parameter defined as

$$
\theta_{c^{n-k} b^{k}}^{(k)} \equiv P_{U_{c^{n-k} b^{k}}^{(k)} \mid U_{c^{n-k}\left[0^{k}: b^{k}\right)}^{(k)}}\left(0 \mid u_{c^{n-k}\left[0^{k}: b^{k}\right)}^{(k)}\right)-P_{U_{c^{n-k} b^{k}}^{(k)} \mid U_{c^{n-k}\left[0^{k}: b^{k}\right)}^{(k)}}\left(1 \mid u_{c^{n-k}\left[0^{k}: b^{k}\right)}^{(k)}\right)
$$

for each $c^{n-k}$ for a given $b^{n} \in\{0,1\}^{n}$. By using (6), (11), and (14), we have the relations

$$
\begin{aligned}
& \theta_{c^{n-k} b^{k-1} 0}^{(k)}=\theta_{c^{n-k} 1 b^{k-1}}^{(k-1)} \theta_{c^{n-k} 0 b^{k-1}}^{(k-1)} \\
& \theta_{c^{n-k} b^{k-1} 1}^{(k)}=\frac{\theta_{c^{n-k} 1 b^{k-1}}^{(k-1)} \mp_{u} \theta_{c^{n-k} 0 b^{k-1}}^{(k-1)}}{1 \mp u} \theta^{(k){ }_{c^{n-k} b^{k}}}
\end{aligned}
$$

where $\mp_{u}$ is the bipolar-binary conversion of $u \equiv u_{c^{n-k} b^{k-1} 0}^{(k)}$. The goal of updateU $\left(\mathrm{U}, n, \mathrm{~b}^{n-1}\right)$ at Line 9 of Algorithm 1 is to compute $u_{c^{n-k} b^{k-1} 0}^{(k)}$ from $u_{\left[0^{n}: b^{n-1} 0\right]}^{(n)}$ by using the relations

$$
\begin{aligned}
& u_{c^{n-k} 0 b^{k-1}}^{(k-1)} \equiv u_{c^{n-k} b^{k-1} 0}^{(k)} \oplus u_{c^{n-k} b^{k-1} 1}^{(k)} \\
& u_{c^{n-k} 1 b^{k-1}}^{(k-1)} \equiv u_{c^{n-k} b^{k-1} 1}^{(k)}
\end{aligned}
$$

that come from (12) and (13), where we assume that $u_{\left[0^{n}: b^{n-1} 0\right]}^{(n)}$ is successfully decoded. It should be noted that (15) and (16 correspond to Lines 5 and 7 of Algorithm 2, respectively, and (17) and (18) correspond to Lines 2 and 3 of Algorithm 3 respectively, where we have relations

$$
\begin{aligned}
\Theta[k]\left[c^{n-k}\right] & =\theta_{c^{n-k} b^{k}}^{(k)} \\
\mathrm{U}[k]\left[c^{n-k}\right]\left[b_{k-1}\right] & =u_{c^{n-k} b^{k}}^{(k)}
\end{aligned}
$$

after completing Lines 3 and 9 of Algorithm 1 respectively. We show (19) and (20) in Section VII-A Furthermore, Line 7 of Algorithm 1 corresponds to the maximum a posteriori probability decision defined as

$$
\widehat{u}_{b^{n}} \equiv \arg \max _{u \in\{0,1\}} P_{U_{b^{n}}^{(n)} \mid U_{\left[0^{n}: b^{n}\right)}^{(n)}}\left(u \mid \widehat{u}_{\left[0^{n}: b^{n}\right)}\right) .
$$

When Algorithm 1 is used for the decoder of polar source code which has access to codeword $u_{\mathcal{I}_{1}}^{(n)}$ and side information vector $y_{\left[0^{n}: 1^{n}\right]}$, we define

$$
P_{U_{c^{n}}^{(0)}}(x) \equiv P_{X_{c^{n}} \mid Y_{c^{n}}}\left(x \mid y_{c^{n}}\right)
$$

for $x \in\{0,1\}$ and obtain the reproduction $\left\{\widehat{x}_{c^{n}}\right\}_{c^{n} \in\left[0^{n}: 1^{n}\right]}$ defined as

$$
\widehat{x}_{c^{n}} \equiv \mathrm{U}[0]\left[c^{n}\right]\left[\widehat{b}_{-1}\right]
$$

where $\widehat{b}_{-1}$ denotes the null string,

When Algorithm 1 is to decode a systematic polar channel code, which has access to channel output vector $y_{\left[0^{n}: 1^{n}\right]}$ and shared vector $u_{\mathcal{I}_{1}}^{(n)}$, we define

$$
P_{U_{c^{n}}^{(0)}}(x) \equiv \frac{P_{Y_{c^{n}} \mid X_{c^{n}}}\left(y_{c^{n}} \mid x\right) P_{X_{c^{n}}}(x)}{\sum_{x^{\prime} \in\{0,1\}} P_{Y_{c^{n}} \mid X_{c^{n}}}\left(y_{c^{n}} \mid x^{\prime}\right) P_{X_{c^{n}}}\left(x^{\prime}\right)}
$$

for given channel distribution $\left\{P_{Y_{c^{n}} \mid X_{c^{n}}}\right\}_{c^{n} \in\left[0^{n}: 1^{n}\right]}$, input distribution $\left\{P_{X_{c^{n}}}\right\}_{c^{n} \in\left[0^{n}: 1^{n}\right]}, x \in\{0,1\}$, and $y_{c^{n}} \in \mathcal{Y}$, This yields a reproduction $\left\{\widehat{x}_{c^{n}}\right\}_{c^{n} \in \mathcal{I}_{0}^{\prime}}$ defined by (22), where $\mathcal{I}_{0}^{\prime}$ is defined by (1).

When Algorithm 1 is used in the decoder of a non-systematic polar channel code, we have to prepare binary variables $\left\{\mathrm{M}\left[b^{n}\right]\right\}_{b^{n} \in \mathcal{I}_{0}}$ and insert

$$
\mathrm{M}\left[\mathrm{b}^{n}\right] \leftarrow \mathrm{U}[n]\left[\mathrm{c}^{0}\right]\left[\mathrm{b}_{n-1}\right]
$$

just after the renewal of $\mathrm{U}[n]\left[\mathrm{c}^{0}\right]\left[\mathrm{b}_{n-1}\right]$ (Line 7 of Algorithm 1 . This yields reproduction $\widehat{u}_{\mathcal{I}_{0}}$ defined as

$$
\widehat{u}_{b^{n}} \equiv \mathrm{M}\left[b^{n}\right] .
$$

Remark 1: When Algorithm 1 is applied to a binary erasure channel, we can assume that $\Theta[\mathrm{k}]\left[\mathrm{c}^{n-\mathrm{k}}\right]$ takes a value in $\{-1,0,1\}$, where $\Theta[0]\left[\mathrm{b}^{n}\right] \leftarrow P_{U_{\mathrm{b}^{n}}^{(0)}}(0)-P_{U_{\mathrm{b}^{n}}^{(0)}}(1)$ in Line 1 of Algorithm 1 can be replaced by

$$
\Theta[0]\left[\mathrm{b}^{n}\right] \leftarrow \begin{cases}1 & \text { if } y_{\mathrm{b}^{n}}=0 \\ 0 & \text { if } y_{\mathrm{b}^{n}} \text { is the erasure symbol } \\ -1 & \text { if } y_{\mathrm{b}^{n}}=1 .\end{cases}
$$

for given channel output $y_{\left[0^{n}: 1^{n}\right]}$. We can improve Algorithm 2 as described in Appendix C. 


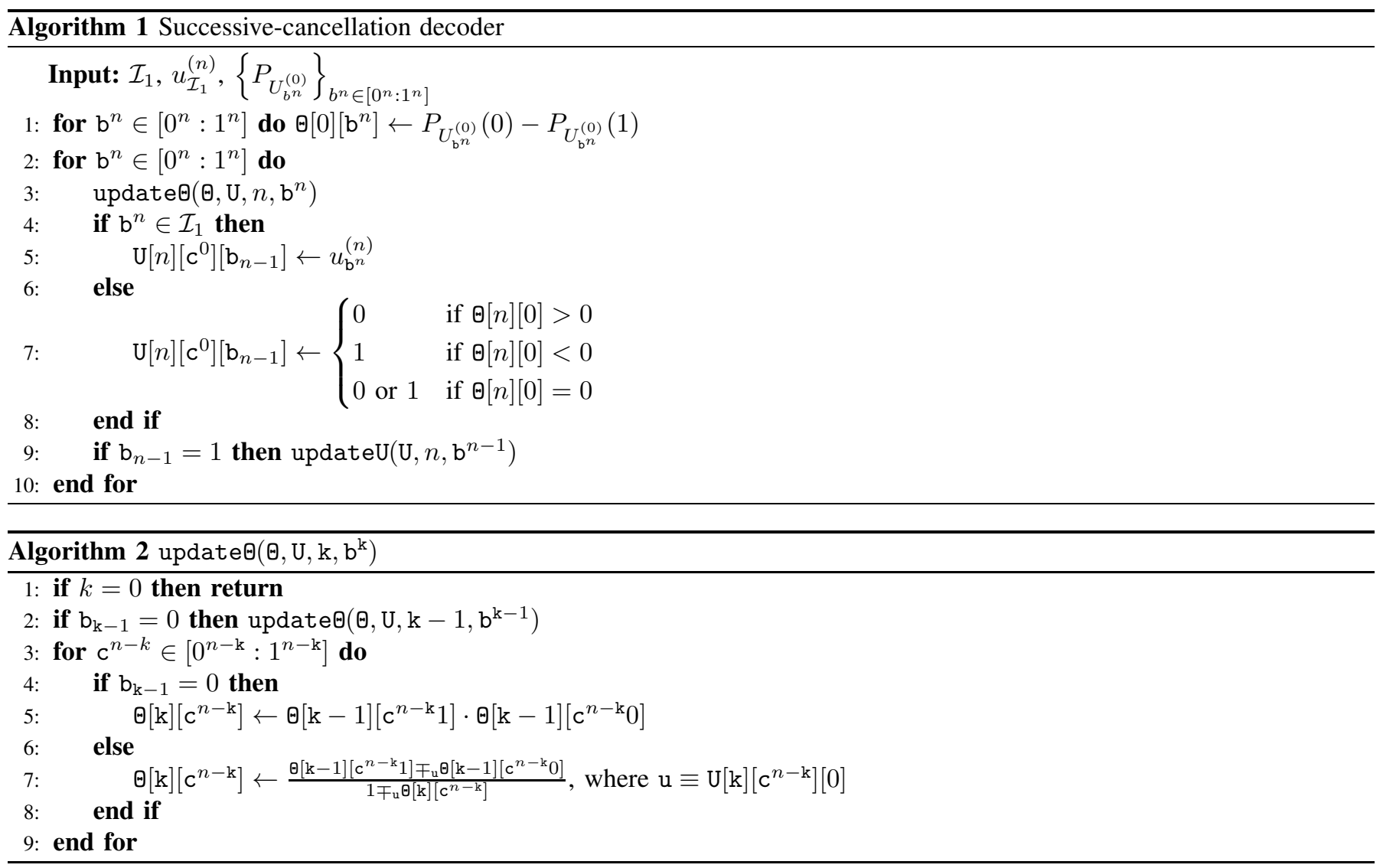

\section{SuCCESSIVE-CANCELlation List Decoding}

This section introduces an algorithm for the successive-cancellation list decoding. It is based on that introduced in [10]. It should be noted that we use a fixed-addressing memory space instead of the stacking memory space approach used in [10]. Since the size of memory space for the computation of conditional probability is around half that used in [10], the time complexity of our algorithm is around half that mentioned in [10].

We assume that Algorithms 249 have access to the number of transforms, $n$, the list size $L$, the frozen bits $u_{\mathcal{I}_{1}}^{(n)}$, and the memory space $\{\Theta[\lambda]\}_{\lambda=0}^{L-1},\{\mathrm{U}[\lambda]\}_{\lambda=0}^{L-1},\{\mathrm{P}[\lambda]\}_{\lambda=0}^{L-1},\{\text { Active }[\lambda]\}_{\lambda=0}^{2 L-1}$, where $\Theta[\lambda]$ and $\mathrm{U}[\lambda]$ are accessed by Algorithms 2 and 3. $\{\mathrm{P}[\lambda]\}_{\lambda=0}^{2 L-1}$ are real number variables, and $\{\text { Active }[\lambda]\}_{\lambda=0}^{2 L-1}$ are binary variables. After Algorithm 4 concludes, the results are stored in $\{\mathrm{U}[\lambda]\}_{\lambda=0}^{L-1}$ and $\{\mathrm{P}[\lambda]\}_{\lambda=0}^{2 L-1}$ satisfying

$$
\begin{aligned}
\frac{\mathrm{P}[\lambda]}{2^{N}} & =\prod_{b^{n} \in\left[0^{n}: 1^{n}\right]} P_{U_{b^{n}}^{(n)} \mid U_{\left[0^{n}: b^{n}\right)}^{(n)}}\left(\widehat{u}_{b^{n}}^{(n)}(\lambda) \mid \widehat{u}_{\left[0^{n}: b^{n}\right)}^{(n)}(\lambda)\right) \\
& =P_{U_{\left[0^{n}: 1^{n}\right]}^{(n)}}\left(\widehat{u}_{\left[0^{n}: 1^{n}\right]}^{(n)}(\lambda)\right)
\end{aligned}
$$

and

$$
\begin{aligned}
\mathrm{U}[\lambda][0]\left[c^{n}\right]\left[b_{-1}\right] & =\widehat{u}_{c^{n}}^{(0)}(\lambda) \\
\widehat{u}_{\left[0^{n}: 1^{n}\right]}^{(n)}(\lambda) & =\widehat{u}_{\left[0^{n}: 1^{n}\right]}^{(0)}(\lambda) G,
\end{aligned}
$$

where $\widehat{u}_{\left[0^{n}: 1^{n}\right]}^{(n)}(\lambda)$ is the $\lambda$-th surviving path. It should be noted that, at Line 5 of Algorithm 7 we select paths $\widehat{u}_{\left[0^{n}: b^{n}\right]}^{(n)}$ that have the $L$ largest probability

$$
\begin{aligned}
\frac{\mathrm{P}[\lambda]}{2^{\left|\left[0^{n}: b^{n}\right]\right|}} & =\prod_{d^{n} \in\left[0^{n}: b^{n}\right]} P_{U_{d^{n}}^{(n)} \mid U_{\left[0^{n}: d^{n}\right)}^{(n)}}\left(\widehat{u}_{d^{n}}^{(n)}(\lambda) \mid \widehat{u}_{\left[0^{n}: d^{n}\right)}^{(n)}(\lambda)\right) \\
& =P_{U_{\left[0^{n}: b^{n}\right]}^{(n)}}\left(\widehat{u}_{\left[0^{n}: b^{n}\right]}^{(n)}(\lambda)\right)
\end{aligned}
$$

where $\widehat{u}_{\left[0^{n}: b^{n}\right]}^{(n)}(\lambda)$ is the $\lambda$-th surviving path. We show (23) and (24) in Section VII-B 


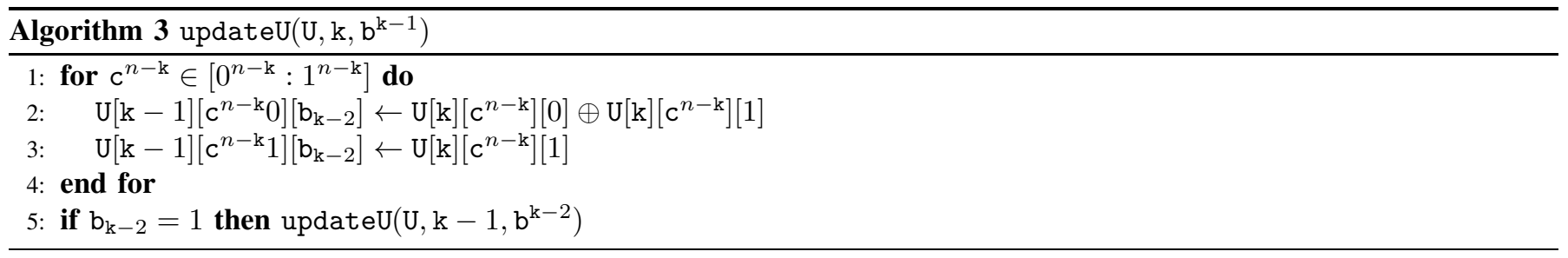

When Algorithm 4 is used in the decoder of polar source code that has access to the codeword $u_{\mathcal{I}_{1}}$ and side information vector $y_{\left[0^{n}: 1^{n}\right]}$, we define $P_{U_{b^{n}}^{(0)}}$ by $(21)$ and obtain reproduction $\left\{\widehat{x}_{c^{n}}(l)\right\}_{c^{n} \in\left[0^{n}: 1^{n}\right]}$ defined as

$$
\widehat{x}_{c^{n}}(l) \equiv \mathrm{U}[l][0]\left[c^{n}\right]\left[b_{-1}\right],
$$

where

$$
l \equiv \arg \max _{\lambda} \mathrm{P}[\lambda]
$$

When the outer parity check function parity generates an extension $s \equiv \operatorname{parity}\left(x^{n}\right)$ to the codeword $u_{\mathcal{I}_{1}}$, the corresponding reproduction is defined as (25) for an $l$ satisfying parity $\left(\left\{\widehat{x}_{c^{n}}(l)\right\}_{c^{n} \in\left[0^{n}: 1^{n}\right]}\right)=\boldsymbol{s}$.

When Algorithm 4 is used in the decoder of systematic polar channel code that has access to channel output vector $y_{\left[0^{n}: 1^{n}\right]}$, and shared vector $u_{\mathcal{I}_{1}}$, we obtain reproduction $\left\{\widehat{x}_{c^{n}}(l)\right\}_{c^{n} \in \mathcal{I}_{0}^{\prime}}$ defined by 25) and 26), where $\mathcal{I}_{0}^{\prime}$ is defined by (11). When we use the outer parity check function parity (e.g. polar code with CRC [10]) with check vector $s$ satisfying $s=\operatorname{parity}\left(x^{n}\right)$ for all channel inputs $x^{n}$, the resulting reproduction $\left\{\widehat{x}_{c^{n}}(l)\right\}_{c^{n} \in \mathcal{I}_{0}^{\prime}}$ is defined as (25) for an $l$ satisfying $\operatorname{parity}\left(\left\{\widehat{x}_{c^{n}}(l)\right\}_{c^{n} \in\left[0^{n}: 1^{n}\right]}\right)=s$.

When Algorithm 4 is used in the decoder of non-systematic polar channel code, we have to prepare binary variables $\left\{\mathrm{M}[\lambda]\left[b^{n}\right]\right\}_{\lambda \in\{0, \ldots L-1\}, b^{n} \in \mathcal{I}_{0}}$ and insert

$$
\begin{aligned}
& \mathrm{M}[\lambda]\left[\mathrm{b}^{n}\right] \leftarrow \mathrm{U}[\lambda][n]\left[\mathrm{c}^{0}\right]\left[\mathrm{b}_{n-1}\right] \\
& \mathrm{M}[\Lambda+\lambda]\left[\mathrm{b}^{n}\right] \leftarrow \mathrm{U}[\Lambda+\lambda][n]\left[\mathrm{c}^{0}\right]\left[\mathrm{b}_{n-1}\right]
\end{aligned}
$$

just after the renewal of $\mathrm{U}[\lambda][n]\left[\mathrm{c}^{0}\right]\left[\mathrm{b}_{n-1}\right]$ and $\mathrm{U}[\Lambda+\lambda][n]\left[\mathrm{c}^{0}\right]\left[\mathrm{b}_{n-1}\right]$, respectively (Lines 5 and 6 of Algorithm 6 , and Lines 8 , 11,15 , and 26 of Algorithm [7). This yields reproduction $\left\{\widehat{u}_{b^{n}}(l)\right\}_{b^{n} \in \mathcal{I}_{0}}$ defined as

$$
\widehat{u}_{b^{n}}(l) \equiv \mathrm{M}[l]\left[b^{n}\right],
$$

where $l$ is defined by (26). When we use the outer parity check function parity (e.g. polar code with CRC [10]) with check vector $s$ satisfying $s=\operatorname{parity}\left(x^{n}\right)$ for all channel input $x^{n}$, the resulting reproduction $\left\{\widehat{u}_{b^{n}}(l)\right\}_{b^{n} \in \mathcal{I}_{0}}$ of the nonsystematic code is defined as (27) for an $l$ such that corresponding channel input $\left\{\widehat{x}_{c^{n}}(l)\right\}_{c^{n} \in\left[0^{n}: 1^{n}\right]}$ defined by (25) satisfies parity $\left(\left\{\widehat{x}_{c^{n}}(l)\right\}_{c^{n} \in\left[0^{n}: 1^{n}\right]}\right)=s$.

For completeness, we introduce an algorithm for Line 5 of Algorithm 7 in Appendix D

Remark 2: Line 17 of Algorithm 4 is unnecessary if we use the infinite precision real number variables. We assumed the use of the finite precision (floating point) real number variables to prevent $\mathrm{P}[\lambda]$ from vanishing as $b^{n}$ increases. We can skip Line 17 of Algorithm 4 and Line 2 of Algorithm 5 while $b^{n} \in \mathcal{I}_{1}$ is satisfied continuously from the beginning $\left(b^{n}=0^{n}\right)$. It should be noted that this type of technique is used in [10, Algorithm 10, Lines 20-25], where this technique is repeated $N n$ times. In contrast, Algorithm 4 uses this technique outside the renewal of parameters $\{\Theta[\lambda]\}_{\lambda=0}^{L-1}$ (Algorithm 2$)$, where magnifyP $(\Lambda)$ is repeated $N$ times.

Remark 3: When we assume that $L$ is a power of $2, \Lambda=L$ is always satisfied at Line 13 of Algorithm 4 Accordingly, we can omit Line 14 of Algorithm 4 and Lines 9-12 of Algorithm 7 because $\Lambda+l \geq L$ is always satisfied.

\section{PROOFS}

\section{A. Proof of (19) and (20)}

Here, we check that we can compute $u_{b^{k} 0}^{(k)}$ from $u_{\left[0^{n}: b^{n}\right)}^{(n)}$. We introduce the following theorems. In the proof of theorems, we write $d^{n}<b^{n}$ when the corresponding integers satisfy the same relation.

Theorem 1: For a given $b^{n} \equiv\left(b_{0}, b_{1}, \ldots, b_{n-1}\right)$, we have

$$
\mathrm{U}[k]\left[c^{n-k}\right]\left[b_{k-1}\right]=u_{c^{n-k} b^{k}}^{(k)}
$$

for all $k$ and $c^{n-k} \in\{0,1\}^{n-k}$ after the operations

$$
\begin{aligned}
& \mathrm{U}[n]\left[c^{0}\right][0] \leftarrow u_{d^{n-1} 0}^{(n)} \\
& \mathrm{U}[n]\left[c^{0}\right][1] \leftarrow u_{d^{n-1} 1}^{(n)}
\end{aligned}
$$



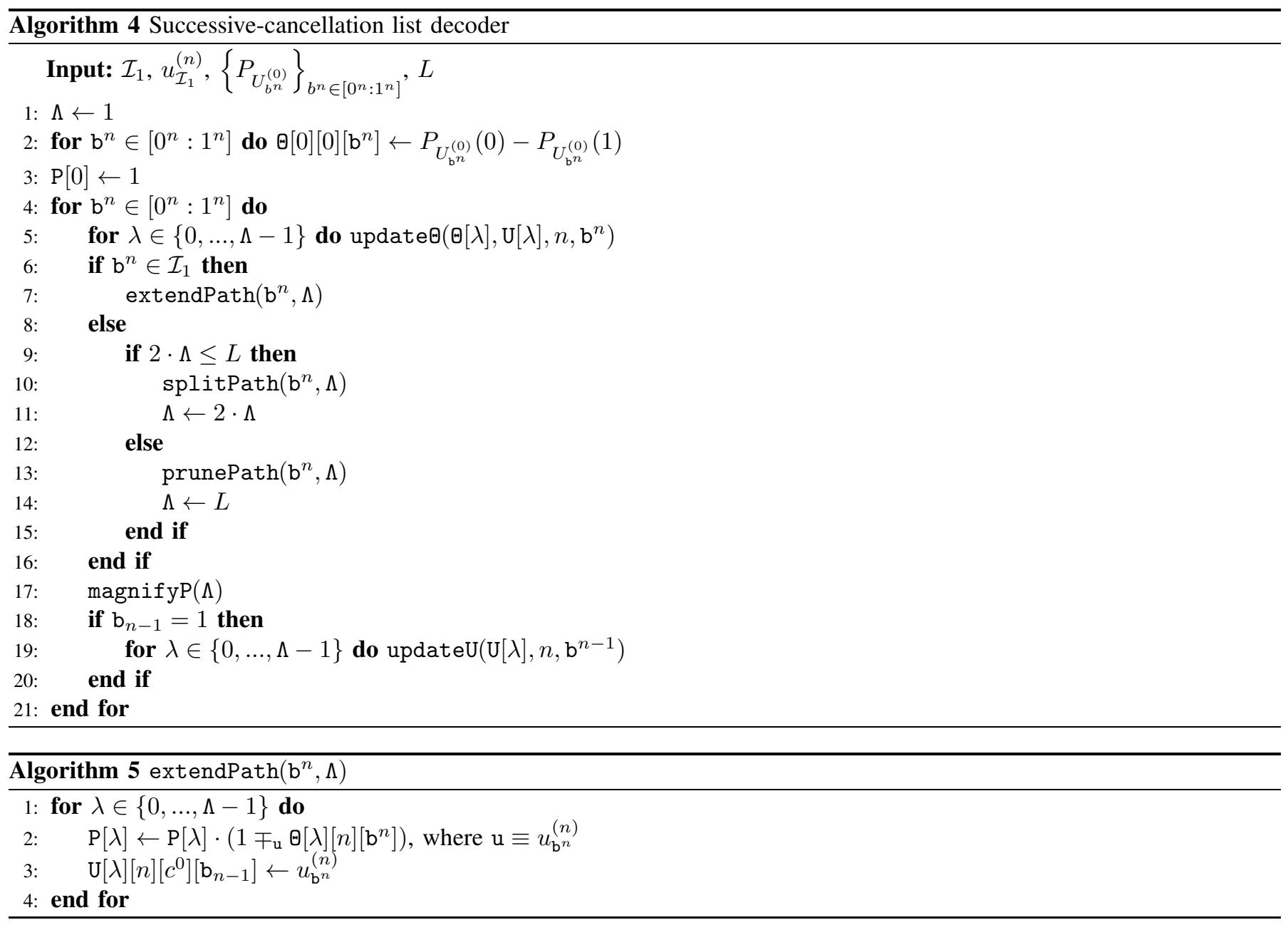

$$
\text { updateU(U, } \left.n, d^{n-1}\right)
$$

employed for each $d^{n-1} \in\left[0^{n}: b^{n-1}\right]$. In particular, after the operations 29]-31 for each $d^{n-1} \in\left[0^{n}: 1^{n-1}\right]$,

$$
\mathrm{U}[0]\left[c^{n}\right]\left[d_{-1}\right]=u_{c^{n}}^{(0)}
$$

for all $c^{n} \in\left[0^{n}: 1^{n}\right]$.

Proof: For a given $b^{n} \in\left[0^{n}: 1^{n}\right]$, we have

$$
\mathrm{U}[n]\left[c^{0}\right]\left[b_{n-1}\right]=u_{b^{n}}^{(n)}=u_{c^{0} b^{n}}^{(n)}
$$

after the operations (29) and (30).

From Line 4 of Algorithm 3, updateU(U, $\left.k, b^{k-1}\right)$ is called only when $\left(b_{k-1}, \ldots, b_{n-1}\right)=1^{n-k+1}$. Let us assume that $\left(b_{k}, \ldots, b_{n-1}\right)=1^{n-k}$. Since $b^{k} 01^{n-k-1}<b^{n}$, we have the fact that updateU $\left(k+1, b^{k}\right)$ is called and $\mathrm{U}[k]\left[c^{n-k-1} 0\right][0]$ and $\mathrm{U}[k]\left[c^{n-k-1} 1\right][0]$ are defined. Here, let us assume that $\mathrm{U}[k]\left[c^{n-k}\right][b]=u_{c^{n-k} b^{k-1} b}^{(k)}$ for all $c^{n-k}$ and $b \in\{0,1\}$. Then we have

$$
\begin{aligned}
\mathrm{U}[k-1]\left[c^{n-k} 0\right]\left[b_{k-2}\right] & =\mathrm{U}[k]\left[c^{n-k}\right][0] \oplus \mathrm{U}[k]\left[c^{n-k}\right][1] \\
& =u_{c^{n-k} b^{k-1} 0}^{(k)} \oplus u_{c^{n-k} b^{k-1} 1}^{(k)} \\
& =u_{c^{n-k} 0 b^{k-1}}^{(k-1)} \oplus u_{c^{n-k} 1 b^{k-1}}^{(k-1)} \oplus u_{c^{n-k} 1 b^{k-1}}^{(k-1)} \\
& =u_{c^{n-k} 0 b^{k-1}}^{(k-1)},
\end{aligned}
$$

where the third equality comes from (17) and (18). In addition, we have

$$
\begin{aligned}
\mathrm{U}[k-1]\left[c^{n-k} 1\right]\left[b_{k-2}\right] & =\mathrm{U}[k]\left[c^{n-k}\right][1] \\
& =u_{c^{n-k} b^{k-1} 1}^{(k)} \\
& =u_{c^{n-k} 1 b^{k-1}}^{(k-1)}
\end{aligned}
$$



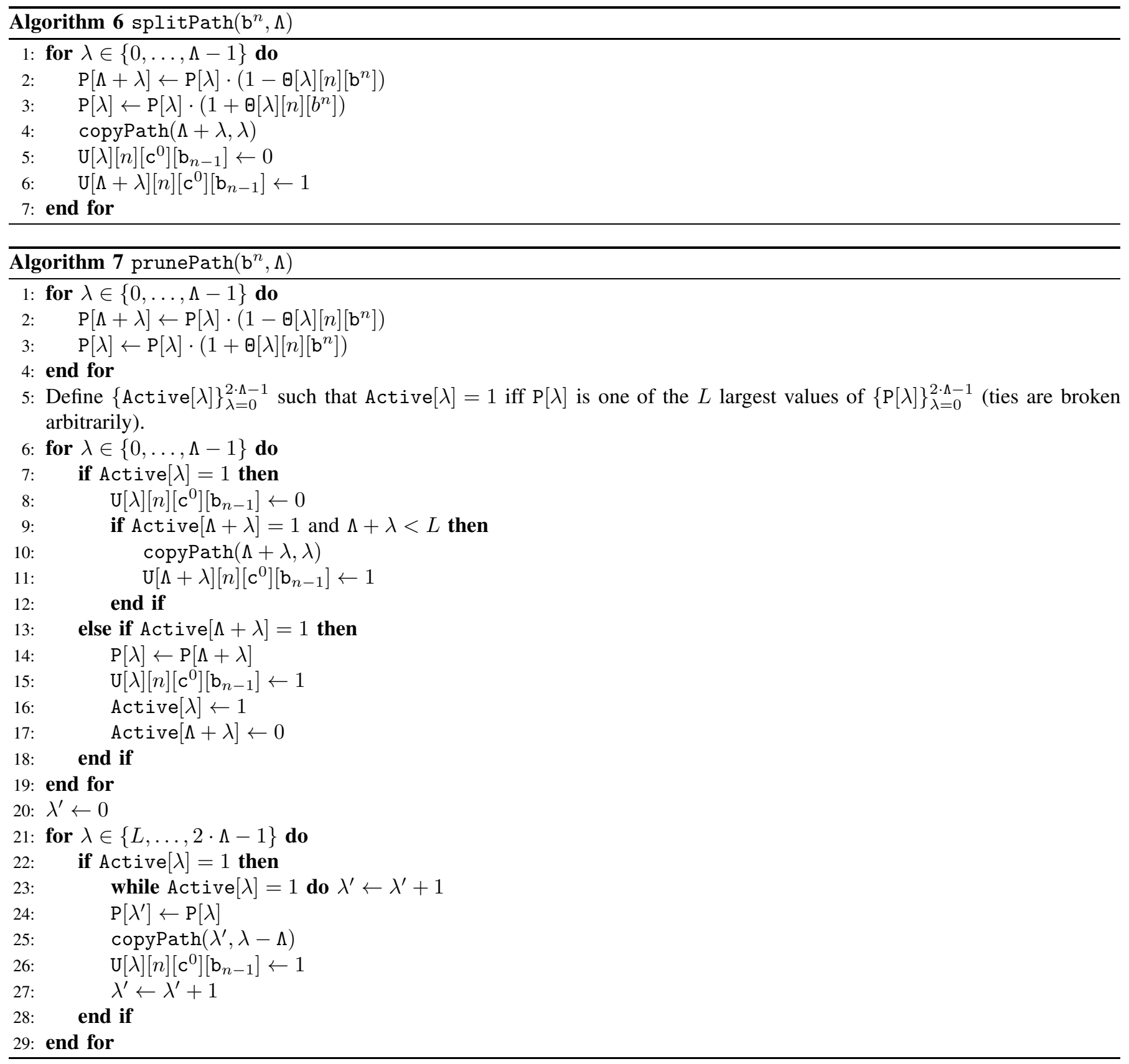

where the last equality comes from $(18)$. The above yields

$$
\mathrm{U}[k-1]\left[c^{n-k+1}\right]\left[b_{k-2}\right]=u_{c^{n-k+1} b^{k-1}}^{(k-1)},
$$

for all $c^{n-k+1}$. Induction yields the relation (28) for all $k$ and $c^{n-k}$ for a given $b^{n}$. By letting $k=0$ and $b^{n}=1^{n}$, we have the fact that updateU $\left(\mathrm{U}, 0, b_{-1}\right)$ is called and $\mathrm{U}[n]\left[c^{n}\right]\left[b_{-1}\right]$ satisfies (32) for all $c^{n}$.

Theorem 2: Assume that

$$
\Theta[0]\left[c^{n}\right]=\theta_{c^{n}}^{(0)}
$$

for all $c^{n} \in\left[0^{n}: 1^{n}\right]$. Then, for a given $b^{n} \equiv\left(b_{0}, b_{1}, \ldots, b_{n-1}\right)$, we have

$$
\Theta[k]\left[c^{n-k}\right]=\theta_{c^{n-k} b^{k}}^{(k)}
$$

for all $k$ and $c^{n-k} \in\{0,1\}^{n-k}$ after the operations

$$
\begin{aligned}
& \text { update } \Theta\left(\Theta, n, d^{n-1}\right) \\
& \mathrm{U}[n]\left[c^{0}\right][0] \leftarrow u_{d^{n-1} 0}^{(n)}
\end{aligned}
$$



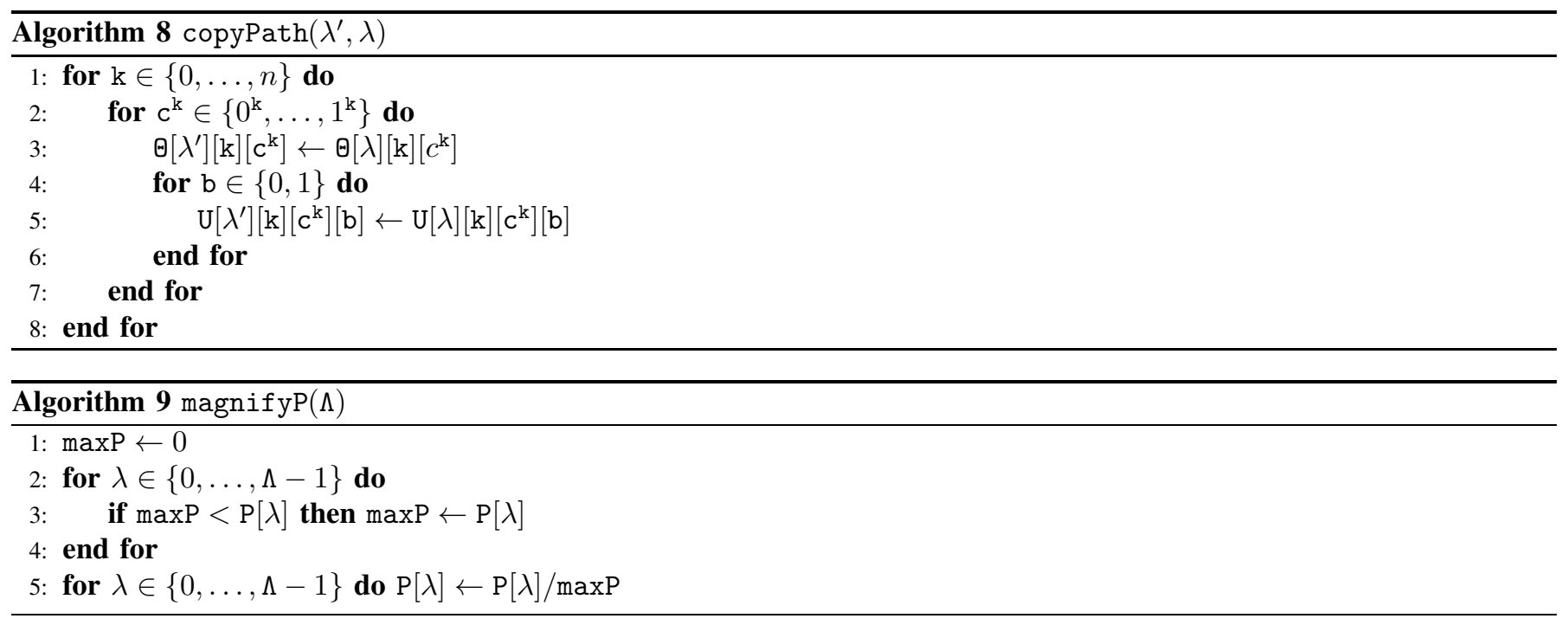

$$
\begin{aligned}
& \mathrm{U}[n]\left[c^{0}\right][1] \leftarrow u_{d^{n-1} 1}^{(n)} \\
& \text { updateU(U, } \left.n, d^{n-1}\right)
\end{aligned}
$$

for each $d^{n-1} \in\left[0^{n}: b^{n-1}\right)$ and

$$
\text { update } \Theta\left(\Theta, n, b^{n-1}\right) \text {. }
$$

Proof: We have the fact that update $\Theta\left(\Theta, k-1, b^{k-1}\right)$ is called only when $\left(b_{k}, \ldots, b_{n-1}\right)=0^{n-k}$. Let $b^{0}$ denote the null string.

Let us assume that (36) is satisfied for all $k \in\{1, \ldots, n-1\}$ and $c^{n-k}$ and

$$
\Theta[k-1]\left[c^{n-k+1}\right]=\theta_{c^{n-k+1} b^{k-1}}^{(k-1)}
$$

for all $c^{n-1}$ and $b^{n}$, where this equality is satisfied when $k=1$ from assumption 35.

Assume that $b_{k-1}=0$. Since $b^{k-2} 0^{n-k+2}<b^{n}$, then update $\Theta\left(\Theta, k-1, b^{k-1}\right)$ is called and

$$
\begin{aligned}
\Theta[k]\left[c^{n-k}\right] & =\Theta[k-1]\left[c^{n-k} 1\right] \cdot \Theta[k-1]\left[c^{n-k} 0\right] \\
& =\theta_{c^{n-k} 1 b^{k-1}}^{(k-1)} \theta_{c^{n-k} 0 b^{k-1}}^{(k-1)} \\
& =\theta_{c^{n-k} b^{k-1} 0}^{(k)}
\end{aligned}
$$

for all $c^{n-1}$, the first equality comes from Line 5 of Algorithm 3 , the second equality comes from (37), and the last equality comes from (15).

Assume that $b_{k-1}=1$. Since $b^{k-2} 0^{n-k+2}<b^{n}$, update $\Theta\left(\Theta, k-1, b^{k-1}\right)$ is called and we have (38). Furthermore, since $b^{k-2} 01^{n-k+1}<b^{n}$, then updateU(U, $\left.k, b^{k-1}\right)$ is called and we have

$$
\mathrm{U}[k]\left[c^{n-k}\right][0]=u_{c^{n-k} b^{k-1} 0}^{(k)}
$$

from Theorem 1 . Then we have

$$
\begin{aligned}
\Theta[k]\left[c^{n-k}\right] & =\frac{\Theta[k-1]\left[c^{n-k} 1\right] \mp_{u} \Theta[k-1]\left[c^{n-k} 0\right]}{1 \mp_{u} \theta_{c^{n-k} b^{k-1} 0}^{(k)}} \\
& =\frac{\theta_{c^{n-k} 1 b^{k-1}}^{(k-1)} \mp_{u} \theta_{c^{n-k} 0 b^{k-1}}^{(k-1)}}{1 \mp_{u} \theta_{c^{n-k} b^{k-1} 0}^{(k)}} \\
& =\theta_{c^{n-k} b^{k-1} 1}^{(k)}
\end{aligned}
$$

for all $c^{n-k}$, where

$$
\begin{aligned}
u & \equiv \mathrm{U}[k]\left[c^{n-k}\right][0] \\
& =u_{c^{n-k} b^{k-1} 0}^{(k)}
\end{aligned}
$$

from (39), where the first equality comes from Line 7 of Algorithm 3 and (38), the second equality comes from (37), and the last equality comes from (16).

From (38) and (40), we have (36) for all $c^{n-1}$ and $b^{n}$ by induction. 


\section{B. Proof of (23) and (24)}

Here, we show (24) by proving the folloing theorem, where (23) is shown by letting $b^{n} \equiv 1^{n}$.

Theorem 3: Let $\widehat{u}_{\left[0^{n}: b^{n}\right]}^{(n)}(\lambda)$ be the $\lambda$-th surviving path after employing one of Algorithms 5-7 (at Line 17 of Algoirthm 4 ). We have

$$
\begin{aligned}
\frac{\mathrm{P}[\lambda]}{2^{\left|\left[0^{n}: b^{n}\right]\right|}} & =\prod_{d^{n} \in\left[0^{n}: b^{n}\right]} P_{U_{d^{n}}^{(n)} \mid U_{\left[0^{n}: d^{n}\right)}^{(n)}}\left(\widehat{u}_{d^{n}}^{(n)}(\lambda) \mid \widehat{u}_{\left[0^{n}: d^{n}\right)}^{(n)}(\lambda)\right) \\
& =P_{U_{\left[0^{n}: b^{n}\right]}^{(n)}}\left(\widehat{u}_{\left[0^{n}: b^{n}\right]}^{(n)}(\lambda)\right) .
\end{aligned}
$$

Proof: After employing one of Algorithms 5 , we have the substitution

$$
P[\lambda] \leftarrow P[\lambda] \cdot\left(1 \mp \widehat{\iota} \Theta[\lambda][n]\left[b^{n}\right]\right)
$$

where

$$
\widehat{u}(\lambda) \equiv \widehat{u}_{b^{n}}^{(n)}(\lambda)
$$

is the $b^{n}$-th symbol of the $\lambda$-th surviving binary path $\widehat{u}_{\left[0^{n}: b^{n}\right]}^{(n)}(\lambda)$. Then we have

$$
\begin{aligned}
\frac{P[\lambda]}{2^{\left|\left[0^{n}: b^{n}\right]\right|}}=\prod_{d^{n} \in\left[0^{n}: b^{n}\right]} \frac{1 \mp \widehat{u}(\lambda) \Theta[\lambda][n]\left[c^{0}\right]}{2} \\
=\prod_{d^{n} \in\left[0^{n}: b^{n}\right]} \frac{1 \mp \widehat{u}(\lambda) \theta_{d^{n}}^{(n)}(\lambda)}{2} \\
=\prod_{d^{n} \in\left[0^{n}: b^{n}\right]} P_{U_{d^{n}}^{(n)} \mid U_{\left[0^{n}: d^{n}\right)}^{(n)}\left(\widehat{u}_{d^{n}}^{(n)}(\lambda) \mid \widehat{u}_{\left[0^{n}: d^{n}\right)}^{(n)}(\lambda)\right)} \\
=P_{U_{\left[0^{n}: b^{n}\right]}^{(n)}\left(\widehat{u}_{\left[0^{n}: b^{n}\right]}^{(n)}(\lambda)\right),}
\end{aligned}
$$

where

$$
\theta_{d^{n}}^{(n)}(\lambda) \equiv P_{U_{d^{n}}^{(n)} \mid U_{\left[0^{n}: d^{n}\right)}^{(n)}}\left(0 \mid \widehat{u}_{\left[0^{n}: d^{n}\right)}^{(n)}(\lambda)\right)-P_{U_{d^{n}}^{(n)} \mid U_{\left[0^{n}: d^{n}\right)}^{(n)}}\left(1 \mid \widehat{u}_{\left[0^{n}: d^{n}\right)}^{(n)}(\lambda)\right)
$$

the first equality is shown by induction, the second equality comes from Theorem 2 , and the third equality comes from (43) and (45).

\section{APPENDIX}

\section{A. Algorithm for Polar Transform}

This section introduces the polar transform defined by (12) and (13). We assume that the following algorithm have access to memory space

$$
\left\{\mathrm{u}[k]\left[b^{n}\right]: \begin{array}{rl}
k & \in\{0,1\} \\
b^{n} & \in\left[0^{n}: 1^{n}\right]
\end{array}\right\}
$$

and function $1 \mathrm{sb}(k)$ outputs the least significant bit of $k$, which equals $k \bmod 2$. The result is stored in $\left\{\mathrm{u}[1 \mathrm{sb}(n)]\left[b^{n}\right]\right\}_{b^{n} \in\left[0^{n}: 1^{n}\right]}$. It should be noted that, from the relation $G=G^{-1}$, we have the fact that $\boldsymbol{u}=\boldsymbol{x} G$ is equivalent to $\boldsymbol{x}=\boldsymbol{u} G$. This implies that we can obtain $\boldsymbol{x}$ from $\boldsymbol{u}$ so that $\boldsymbol{x}=\boldsymbol{u} G^{-1}=\boldsymbol{u} G$ is satisfied.

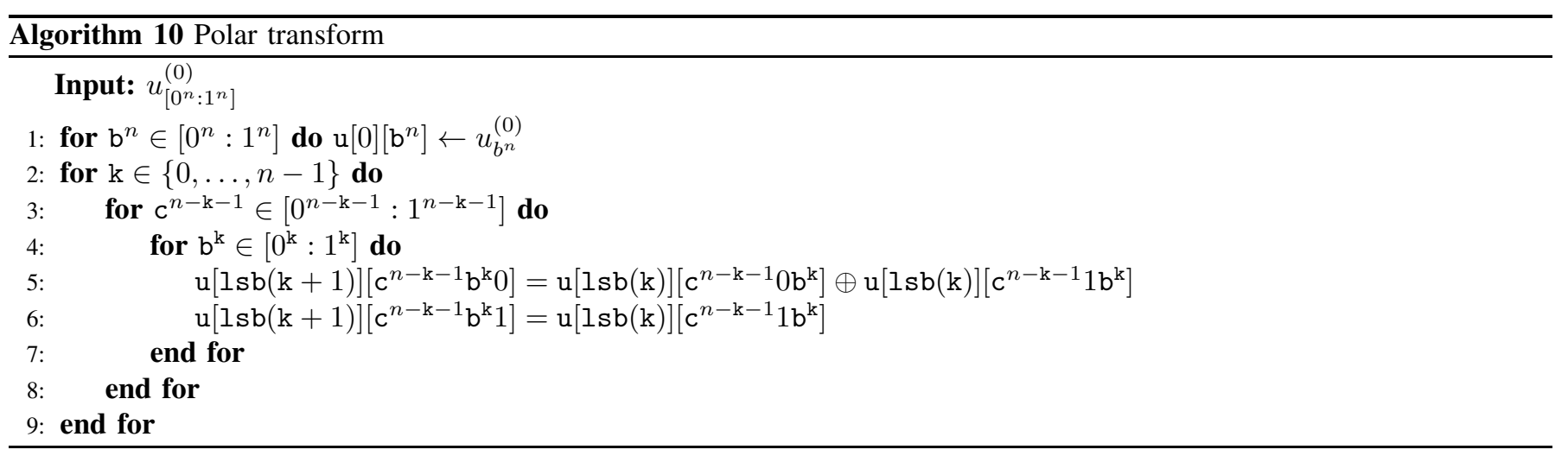




\section{B. Algorithm for Systematic Channel Encoder}

For completeness, we introduce an algorithm for the systematic encoder of a polar channel code based on [3].

The following algorithm finds $x_{\mathcal{I}_{1}^{\prime}}$ from $x_{\mathcal{I}_{0}^{\prime}}$ and $u_{\mathcal{I}_{1}}$ such that there is $u_{\mathcal{I}_{0}}$ satisfying $\left(x_{\mathcal{I}_{1}^{\prime}}, x_{\mathcal{I}_{0}^{\prime}}\right)=\left(u_{\mathcal{I}_{1}}, u_{\mathcal{I}_{0}}\right) G$, where the elements in $\left(x_{\mathcal{I}_{1}^{\prime}}, x_{\mathcal{I}_{0}^{\prime}}\right)$ and $\left(u_{\mathcal{I}_{1}}, u_{\mathcal{I}_{0}}\right)$ are sorted in index order before operating $G$. It should be noted that $\left(x_{\mathcal{I}_{1}^{\prime}}, x_{\mathcal{I}_{0}^{\prime}}\right)=$ $\left(u_{\mathcal{I}_{1}}, u_{\mathcal{I}_{0}}\right) G$ is equivalent to $\left(x_{\mathcal{I}_{1}^{\prime}}, x_{\mathcal{I}_{0}^{\prime}}\right) G=\left(u_{\mathcal{I}_{1}}, u_{\mathcal{I}_{0}}\right)$. We assume that the following algorithms have access to $\mathcal{I}_{1}$ and memory spaces

$$
\begin{aligned}
& \left\{\mathrm{X}\left[b^{n}\right]: b^{n} \in\left[0^{n}: 1^{n}\right]\right\} \\
& \left\{\mathrm{V}\left[b^{n}\right]: b^{n} \in\left[0^{n}: 1^{n}\right]\right\} .
\end{aligned}
$$

The result is stored in $\left\{\mathrm{X}\left[b^{n}\right]\right\}_{b^{n} \in\left[0^{n}: 1^{n}\right]}$, which is also used to refer to the value $u_{\mathcal{I}_{1}}$ in Algorithm 12 For a given $b^{n} \equiv$ $\left(b_{0}, \ldots, b_{n-1}\right)$, let $\operatorname{br}\left(b^{n}\right)$ be defined as

$$
\operatorname{br}\left(b^{n}\right) \equiv\left(b_{n-1}, \ldots, b_{0}\right)
$$
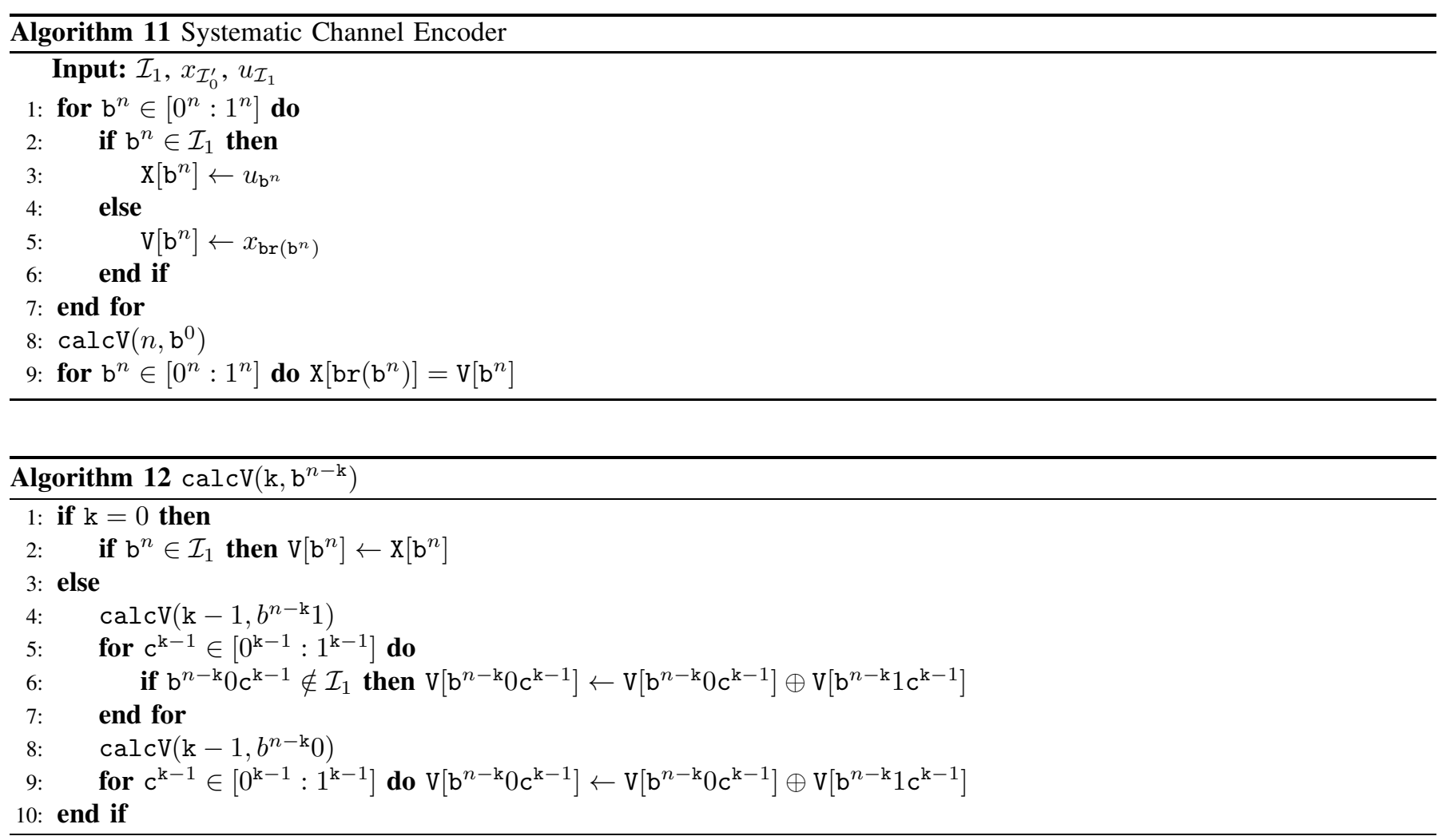

\section{Improvement of Algorithm 2 by Assuming $\Theta[k]\left[c^{n-k}\right] \in\{-1,0,1\}$}

We introduce an improvement for Algorithm 2 by assuming $\Theta[k]\left[c^{n-k}\right] \in\{-1,0,1\}$.

For simplicity, we assume that $\Theta[k]\left[c^{n-k}\right]$ is represented by a 3-bit signed integer consisting of a sign bit and two bits representing an absolute value.

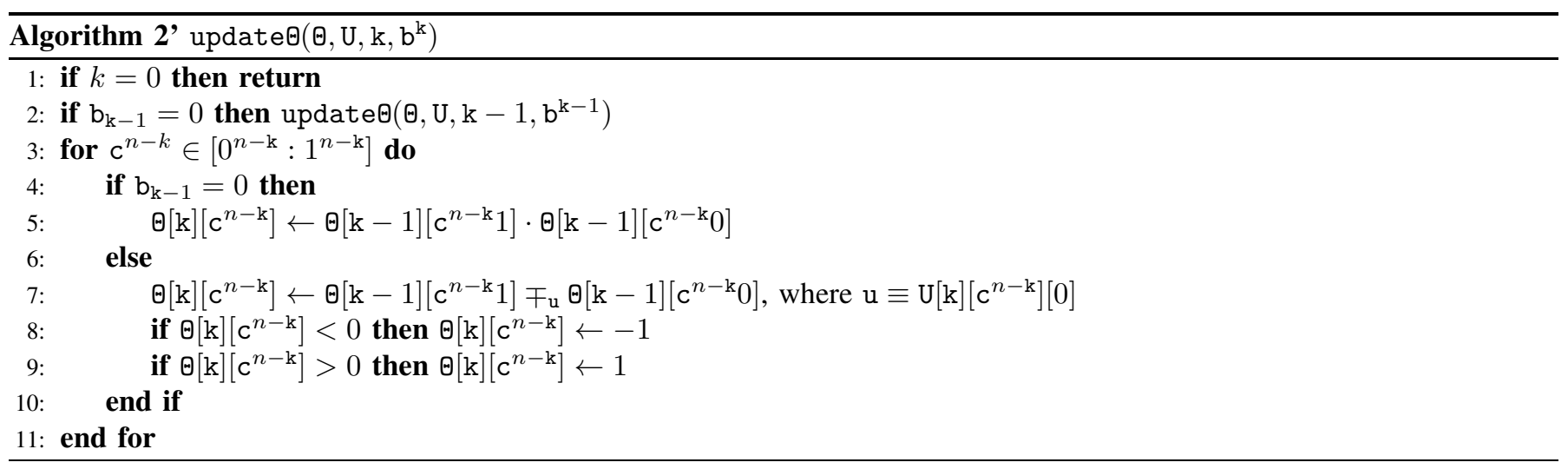




\section{Algorithm for Line 5 of Algorithm 7}

We can implement Line 5 of $\operatorname{Algorithm~} 7$ by markPath $(\Lambda)$ defined as Algorithm 13

We assume that Algorithms 13 16 can access the memory space $\{\mathrm{P}[\lambda]\}_{\lambda=0}^{2 L-1},\{\text { Index }[\lambda]\}_{\lambda=0}^{2 L-1}$, and $\{\text { Active }[\lambda]\}_{\lambda=0}^{2 L-1}$, where Index $[\lambda] \in\{0, \ldots 2 L-1\}$ is an integer variable. The result is stored in $\{\text { Active }[\lambda]\}_{\lambda=0}^{2 \Lambda-1}$.
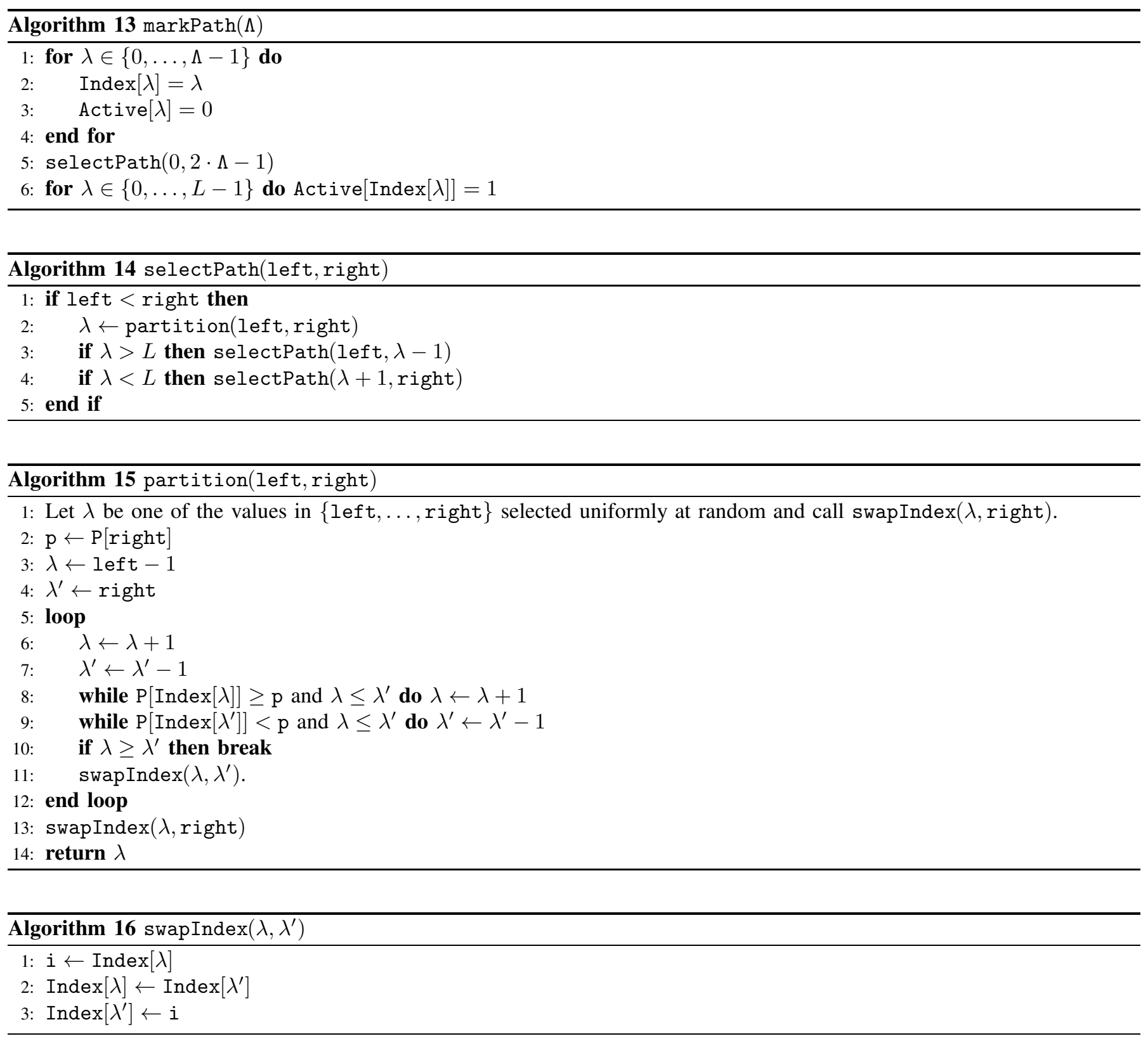

Remark 4: As mentioned in [10], we can simply sort $\{\operatorname{Index}[\lambda]\}_{\lambda=0}^{\Lambda-1}$ so that $\mathrm{P}[$ Index $[0]] \geq \mathrm{P}[\operatorname{Index}[1]] \geq \cdots \geq \mathrm{P}[$ Index $[\Lambda-$ 1]] instead of calling selectPath $(0,2 \cdot \Lambda-1)$ at Line 5 of Algorithm 13 Although the time complexity of sorting is $O(\Lambda \log \Lambda)$, it could be faster than selectPath $(0,2 \cdot \Lambda-1)$ when $\Lambda$ is small.

Remark 5: Line 1 of Algorithm 15, which can be omitted, guarantees that the average time complexity of $\operatorname{selectPath}(0,2$. $\Lambda-1)$ is $O(\Lambda)$. We can replace this line by selecting the index corresponding to the median of $\{\mathrm{P}[\lambda]\}_{\lambda=1 \mathrm{eft}}^{\text {right }}$ to guarantee worst-case time complexity $O(\Lambda)$ (see [4]).

\section{REFERENCES}

[1] E. Arıkan, "Channel polarization: a method for constructing capacity-achieving codes for symmetric binary-input memoryless channels," IEEE Trans. Inform. Theory, vol. IT-55, no. 7, pp. 3051-3073, Jul. 2009.

[2] E. Arıkan, "Source polarization," Proc. 2010 IEEE Int. Symp. Inform. Theory, Austin, U.S.A., June 13-18, 2010, pp. 899-903.

[3] E. Arıkan, "Systematic polar coding," IEEE Communications Letters, vol. 15, no. 8, pp. 860-862, Aug. 2010.

[4] M. Blum, R. W. Floyd, V. Pratt, R. L. Rivest, and R. E. Tarjan, "Time bounds for selection," J. Computer and System Sciences, vol. 7, no. 4, pp. 448-461, 1973. 
[5] R. Mori and T. Tanaka, "Source and channel polarization over finite fields and Reed-Solomon matrices," IEEE Trans. Inform. Theory, vol. IT-60, no. 5, pp. 2720-2736, May 2014.

[6] J. Muramatsu, "Successive-cancellation decoding of linear source code," Proceedings of the 2019 IEEE Information Theory Workshop, Visby, Sweden, Aug. 25-28, 2019. Extended version available at arXiv:1903.11787[ cs.IT], 2019.

[7] J. Muramatsu, "Binary polar codes based on bit error probability," in preparation, 2021.

[8] E. Şaşoğlu, "Polarization and polar codes," Fund. Trends Commun. Inf. Theory, vol. 8, no. 4, pp. 259-381, Oct. 2012.

[9] I. Tal and A. Vardy, "How to construct polar codes," IEEE Trans. Inform. Theory, vol. IT-59, no. 10, pp. 6563-6582, Oct. 2013.

[10] I. Tal and A. Vardy, "List decoding of polar codes," IEEE Trans. Inform. Theory, vol. IT-61, no. 5, pp. 2213-2226, May. 2015. 